\title{
Studies of Be-Fe Compounds Synthesized by Laser Irradiation
}

\author{
A. Amulevicius ${ }^{a}, \mathrm{~K} \cdot$ Mazeika $^{a}, \mathrm{C} \cdot \mathrm{Sipavicius}^{a}$ \\ AND A. BALTUSNIKAS ${ }^{b}$ \\ ${ }^{a}$ Institute of Physics, Savanoriu 231, 02300, Vilnius, Lithuania \\ ${ }^{b}$ Kaunas University of Technology, Donelaicio 73, 44029, Kaunas, Lithuania
}

(Received December 3, 2004; revised version April 7, 2005)

\begin{abstract}
The $\mathrm{FeBe}_{2,5,11}$ compounds and the $\alpha$ - $\mathrm{Fe}(\mathrm{Be})$ solid solution of $\mathrm{Be}_{x} \mathrm{Fe}_{1-x}$ system were synthesized by laser irradiation from a powder mixture. The dependence of the phase structure on the initial sample composition $(x=0.1-0.95)$ was studied. The phases were determined and studied by means of Mössbauer spectroscopy and X-ray diffraction.
\end{abstract}

PACS numbers: 61.18.Fs, 61.10.-i, 61.80.Ba

\section{Introduction}

Beryllium as a light metal has peculiar properties suitable for some future nuclear or space applications [1]. Because of this interest, beryllium-coated iron foils were studied $[2,3]$. Despite some advance over the last century, the knowledge about alloying of beryllium with other elements is still very limited.

Laser application offers additional possibilities of material synthesis. It is suitable for processing and alloying the surface or thin layers. In this article the study of formation of solid solution and compounds of the Be-Fe system, when a thin powder layer is affected by laser irradiation, is presented. Laser alloying of iron with other light elements, forming $\mathrm{Fe}-\mathrm{C}$ and $\mathrm{Fe}-\mathrm{B}$ system compounds, has been already studied in Refs. $[4,5]$. There are differences and similarities between these systems. Boron and beryllium alloyed with iron form a lot of compounds. $\mathrm{Fe}-\mathrm{Be}$ system has a broad region of beryllium supersaturated solid solution in iron and beryllium-rich compounds - $\mathrm{FeBe}_{2,5,11}$ and probably $\mathrm{Fe}_{3} \mathrm{Be}$. The systems with many possible compounds are interesting and favourable materials for studying the distribution of constituents during a short pulse of laser irradiation. 


\section{Experimental details}

The samples were synthesized from a mixture of selected amounts of $\mathrm{Fe}$ and Be powder. The average dimension of Be powder particles was $(230 \pm 100) \mu \mathrm{m}$. The particles of iron were much smaller, 1-4 $\mu \mathrm{m}$. A powder mixture with some glue added was placed on the massive $\mathrm{Cu}$ substrate and flattened. The thickness of a mixture layer was $\approx 57 \mathrm{mg} \mathrm{cm}^{-2}$. For the irradiation, the Nd:YAG laser of $1.06 \mu \mathrm{m}$ wavelength was used. The pulses were of $2 \mathrm{~ms}$ duration and of $150 \mathrm{~Hz}$ repetition frequency. The average power of the laser was $250 \mathrm{~W}$. The diameter of the laser beam spot on the sample surface was approximately $1 \mathrm{~mm}$. The velocity of a scan of $1 \mathrm{~mm} \mathrm{~s}^{-1}$ was chosen so that the same sample area was irradiated by approximately 150 pulses.

The synthesized samples were studied by means of Mössbauer spectroscopy using the ${ }^{57} \mathrm{Co}(\mathrm{Rh})$ source. The phase structure of the samples was mainly defined by Mössbauer spectroscopy. The X-ray diffraction studies using $\mathrm{Cu} K_{\alpha}$ radiation allowed us to obtain the additional information on the phases very rich in beryllium (Fig. 1).

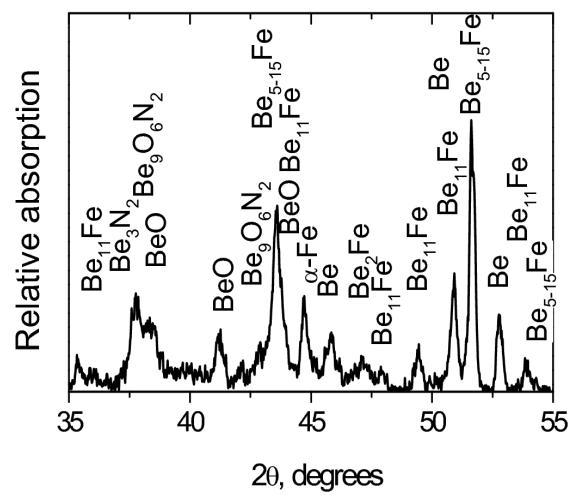

Fig. 1. X-ray diffraction spectra for the $\mathrm{Fe}_{7.5} \mathrm{Be}_{92.5}$ sample.

Typical Mössbauer spectra are shown in Fig. 2a. The spectra are measured at room temperature, except the additional spectrum of the sample of $\mathrm{Be}_{0.77} \mathrm{Fe}_{0.23}$ composition, which is measured at a temperature of $125 \mathrm{~K}$ (cf. Fig. 3a). As the samples consist of many ferromagnetic and paramagnetic phases, most of them of nonstoichiometric composition, one of the best methods of the spectra analysis is to use the distributions of hyperfine parameters.

The distributions of hyperfine parameters are found by the linear least square fitting procedure, which is based on the singular value decomposition (SVD) method [6]. The SVD method is favourable for solving the problem with many parameters because it allows finding a stable solution even if the functions used for the description are very similar. The sets of sextets $f_{1}$ and singlets $f_{2}$, 

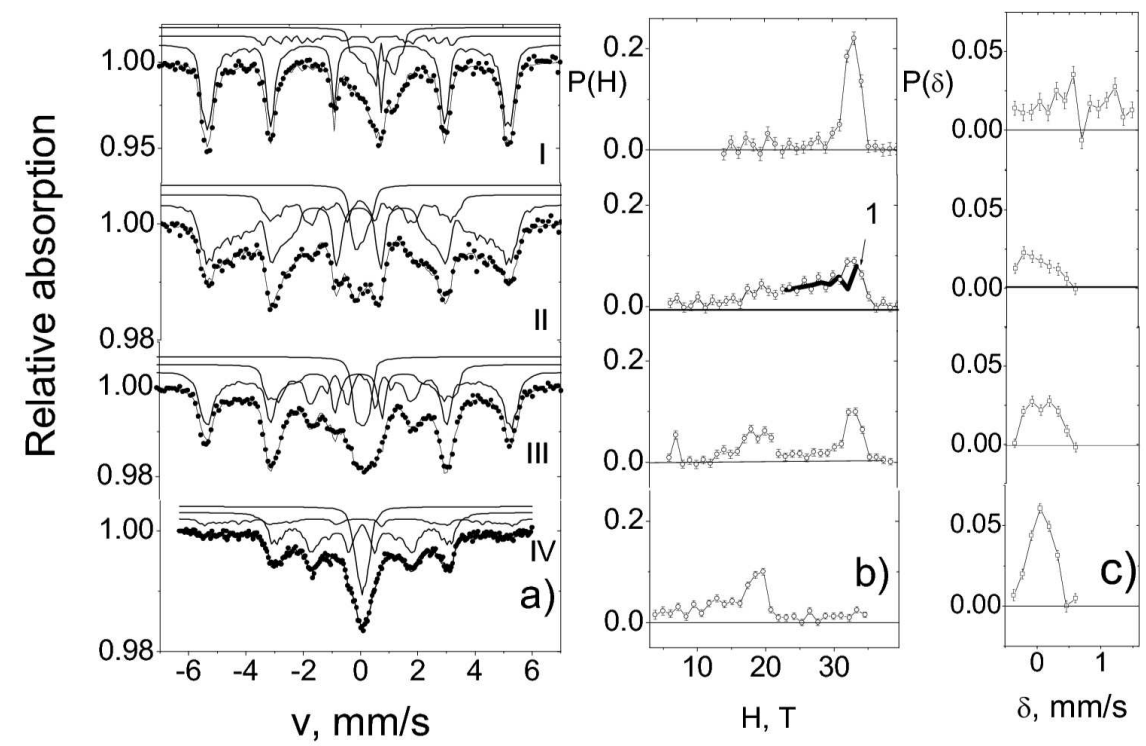

Fig. 2. Mössbauer spectra (a) and their decomposition to the hyperfine magnetic field $P(H)$ (b) and isomer shift $P(\delta)$ (c) distributions of $\mathrm{Be}_{x} \mathrm{Fe}_{1-x}$ samples synthesized by the laser: $\mathrm{I}-x=0.25$, II $-x=0.5$, III $-x=0.7, \mathrm{IV}-x=0.77$ at room temperature. 1 - theoretical $P(H)$ distribution calculated for the uniform distribution of the Be concentration in $\alpha-\mathrm{Fe}(\mathrm{Be})$ solution.

$$
f(v)=\sum_{i} P\left(H_{i}\right) f_{i}\left(H_{i}, \delta_{i}, v\right)+\sum_{i} P\left(\delta_{i}\right) f_{2}\left(\delta_{i}, v\right),
$$

are fitted respectively to ferromagnetic and paramagnetic parts of experimental spectra, where $v$ is the velocity. Thus the hyperfine magnetic field distribution $P(H)$ together with the isomer shift distribution $P(\delta)$ are found. To obtain the quality distributions the number of parameters used in the description should be much less than the number of spectra points [7]. Two sets of sextets of the $0.19 \mathrm{~mm} \mathrm{~s}^{-1}$ linewidth with the hyperfine magnetic field step $\Delta H \approx 1 \mathrm{~T}$ are used to describe the ferromagnetic part of Mössbauer spectra (Fig. 2b). The first set describes $\alpha-\mathrm{Fe}(\mathrm{Be})$ solid solution. For this solution, the hyperfine magnetic field varies within 23-38 T. For the second set of sextets, which describes $\mathrm{FeBe}_{2}$ phase, the hyperfine magnetic field varies within 5-22 T. The isomer shifts $\delta$ for the sets of sextets are determined iteratively by the usual nonlinear least square fitting method using the separate linear isomer shift dependence

$$
\delta_{i}=\delta_{1}+\operatorname{const}\left(H_{i}-H_{1}\right)
$$

for each set.

To take into account the paramagnetic part of Mössbauer spectra in the analysis, the isomer shift distribution $P(\delta)$ (Fig. 2c) is added. It describes beryllium-rich compounds $\mathrm{FeBe}_{5,11}$ (isomer shift range $-0.4 \mathrm{~mm} \mathrm{~s}^{-1}<\delta<0.6 \mathrm{~mm} \mathrm{~s}^{-1}$ ) 
and, for iron-rich samples, the products of iron reaction with atmosphere (isomer shift range $-0.4 \mathrm{~mm} \mathrm{~s}^{-1}<\delta<1.5 \mathrm{~mm} \mathrm{~s}^{-1}$ ). The application of the isomer shift distribution instead of separate doublets or singlets is favourable here because it does not require preliminary data about the compounds. The phases obtained when iron reacts with ambient air are not in the scope of this study. They are most probably very small particles of iron oxides. It is evident that the isomer shifts in the range $0.6 \mathrm{~mm} \mathrm{~s}^{-1}<\delta<1.5 \mathrm{~mm} \mathrm{~s}^{-1}$ (Fig. 2c) could not be attributed to iron-beryllium compounds. In this case, only the large quadrupole splitting, which is characteristic of iron oxides, can explain the experimental isomer shifts for iron-rich $\mathrm{Be}_{x} \mathrm{Fe}_{1-x}$ samples. Only a small amount of beryllium-rich $\mathrm{FeBe}_{5,11}$ compounds could be obtained when $x<0.25$. On the basis of these assumptions the paramagnetic part of $\mathrm{Be}_{x} \mathrm{Fe}_{1-x}$ samples in the composition range $0<x<0.25$ is mainly attributed to iron in the phases which are formed due to iron reaction with atmosphere.

Though the distributions of hyperfine parameters are useful for the evaluation of amounts of the phases and the qualitative analysis of the phases, they are not suitable for the quantitative determination of the percentage of beryllium in $\mathrm{FeBe}_{2+\theta}$. Therefore, the selected Mössbauer spectra are decomposed into alternative sets of subspectra. The Mössbauer spectra of $\mathrm{Be}_{0.77} \mathrm{Fe}_{0.23}$ are decomposed into a set of subspectra for $\mathrm{FeBe}_{2+\theta}$ phase and a doublet for paramagnetic phases (Fig. 3a). The values of the hyperfine magnetic field of the sextets used for $\mathrm{FeBe}_{2+\theta}$ phase are presented in Table I. The paramagnetic parts of $\mathrm{Be}_{0.9} \mathrm{Fe}_{0.1}$ and $\mathrm{Be}_{0.95} \mathrm{Fe}_{0.05}$ spectra, measured at a low velocity (Fig. 3b), are successfully de-

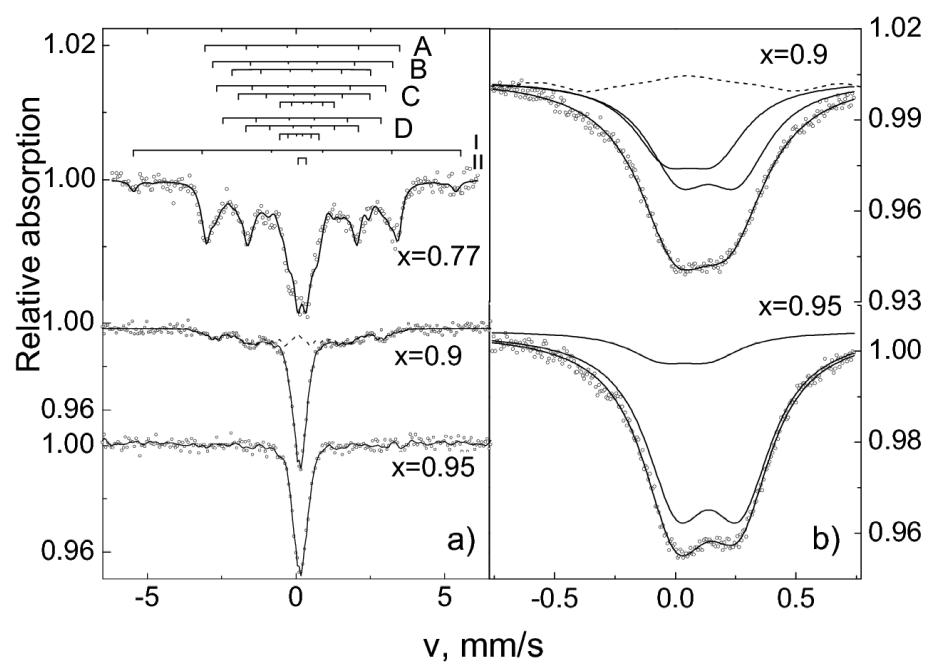

Fig. 3. Mössbauer spectra of $\mathrm{Be}_{x} \mathrm{Fe}_{1-x}(x=0.77,0.9$, and 0.95) samples and their description. The subspectra: (a): I $-\alpha$-Fe(Be), II - paramagnetic phases, $A, B, C, D$ - subspectra of $\mathrm{FeBe}_{2+\theta}$ (Table I); (b): solid lines - doublets; (a) and (b) dashed lines - inner lines of $\mathrm{FeBe}_{2+\theta}$. 
scribed by two doublets, including, for the spectrum of $\mathrm{Be}_{0.9} \mathrm{Fe}_{0.1}$, the inner lines of sextets of $\mathrm{FeBe}_{2+\theta}$ compound, the form which is based on the results of $P(H)$ analysis (Fig. 3a).

TABLE I

The Mössbauer spectra description data for $\mathrm{FeBe}_{2+\theta}$ compound of $\mathrm{Be}_{0.77} \mathrm{Fe}_{0.23}$ sample. $A, B, C, D$ are the regions of different stoichiometry. Hyperfine magnetic fields $H$ and their relative probability $P$ correspond to subspectra of iron atoms having $m$ iron atom first neighbours.

\begin{tabular}{c|c|c|c|c|c|c|c|c|c|c}
\hline \hline \multicolumn{2}{c|}{ Region } & $A$ & \multicolumn{2}{c|}{$B$} & \multicolumn{3}{c|}{$C$} & \multicolumn{3}{c}{$D$} \\
\hline \multicolumn{2}{c|}{$m$} & 4 & 4 & 3 & 4 & 3 & 2 & 4 & 3 & 2 \\
\hline$H, \mathrm{~T}$ & $125 \mathrm{~K}$ & 20.3 & 18.8 & 14.5 & 17.5 & 13.7 & 5.6 & 16.5 & 11.6 & 4.1 \\
& $293 \mathrm{~K}$ & 19.6 & 17.8 & 13.7 & 16.4 & 12.9 & 5.3 & 14.7 & 11.3 & 3.9 \\
$P, \%$ & $125 \mathrm{~K}$ & $34 \pm 5$ & $13 \pm 3$ & $7 \pm 3$ & $8 \pm 3$ & $10 \pm 4$ & $8 \pm 4$ & $6 \pm 3$ & $7 \pm 3$ & $7 \pm 4$ \\
& $293 \mathrm{~K}$ & $31 \pm 4$ & $17 \pm 4$ & $5 \pm 3$ & $9 \pm 4$ & $5 \pm 4$ & $7 \pm 4$ & $7 \pm 3$ & $11 \pm 4$ & $8 \pm 4$ \\
\multicolumn{1}{c|}{$\theta$} & 0 & \multicolumn{2}{c|}{0.33} & \multicolumn{3}{c}{0.63} & & \multicolumn{3}{c}{$\approx 1$}
\end{tabular}

Taking into account a large thickness of samples with a large percentage of iron for Mössbauer spectroscopy, the correction of phase amounts is made according to the calculated dependences of an area of lines on the thickness of the sample. This correction is significant for $\alpha$ - $\mathrm{Fe}(\mathrm{Be})$ solution of the samples containing more than 50 at.\% of Fe.

\section{Modelling of phase structure}

The phase structure in the samples after laser processing is determined by means of Mössbauer spectroscopy. To explain the obtained phase amounts, the distribution of iron and beryllium atoms in the melt during laser irradiation should be known. As the duration of a laser pulse is $2 \mathrm{~ms}$, the entire time of laser processing for each area (approximately 150 pulses) of the samples is $\approx 0.3 \mathrm{~s}$. It is a very short time compared to processing time in the conventional metallurgy, therefore high temperatures, even reaching the boiling point at the surface of samples, are needed for synthesis of compounds from a powder mixture.

From the appearance of iron-rich samples it is evident that they were molten. In beryllium-rich samples the powder is also agglomerated, and at least a partly molten state during laser processing should be achieved. Nevertheless, it is evident that for iron-rich samples the temperatures were higher than those for beryllium-rich ones.

Another confirmation of sufficiently high temperature in the samples is the phase structure (Fig. 4). In iron-rich samples, temperatures higher than the melting-point in the whole sample volume are achieved. The surface temperatures can even reach the boiling point. As a consequence of high temperature 
in the $\mathrm{Be}_{x} \mathrm{Fe}_{1-x}$ samples, in the composition range $0<x<0.4$, a considerable amount of the products of iron reaction with atmosphere is formed (Fig. 4). As the samples melt, the concentrations of beryllium and iron atoms in iron-beryllium phases (for atomic concentration $c_{\mathrm{Be}}+c_{\mathrm{Fe}}=1$ ) during laser processing are determined by processes in the liquid metal when not only diffusion, but also convection are important. Due to the complexity of the processes we will consider only the efficient diffusion which takes into account all such processes. Usually, the diffusion for a binary system is described by the equation of the mutual diffusion

$$
\frac{\partial c_{i}}{\partial t}=\nabla\left(\left(D_{\mathrm{Be}} c_{\mathrm{Fe}}+D_{\mathrm{Fe}} c_{\mathrm{Be}}\right) \nabla c_{i}\right)
$$

where the mutual diffusion coefficient of beryllium in iron $D_{\mathrm{Be}}$ and that of iron in beryllium $D_{\mathrm{Fe}}$ are different. There are not many data reported on the diffusion coefficients for liquid metals. When such data can be found, they are rather inaccurate, which is mainly related to the influence of buoyancy driven forces [8].

The aim of numerical modelling of diffusion is to show that the phase structure can be described by the efficient mutual diffusion of constituent atoms in the samples. For the simplicity, a one-dimensional equation is used and the diffusion is modelled in the spherical coordinates by

$$
\begin{aligned}
\frac{\partial c_{\mathrm{Be}}}{\partial t} & =\left(D_{\mathrm{Fe}}-D_{\mathrm{Be}}\right)\left(\frac{\partial c_{\mathrm{Be}}}{\partial r}\right)^{2} \\
& +\left[D_{\mathrm{Be}}+\left(D_{\mathrm{Fe}}-D_{\mathrm{Be}}\right) c_{\mathrm{Be}}\right] \frac{1}{r^{2}} \frac{\partial}{\partial r}\left(r^{2} \frac{\partial c_{\mathrm{Be}}}{\partial r}\right) .
\end{aligned}
$$

In the one-dimensional model it is assumed that larger beryllium particles are surrounded by the iron layer of such thickness that the volumes of different materials correspond to the initial composition. The average radius of beryllium particles is $115 \pm 50 \mu \mathrm{m}$. The size distribution of beryllium particles is included into the model according to normal law. The concentrations obtained by numerical solving of Eq. (4) are used to calculate the amounts of phases that are fitted to the experimental results. The model should also take into account the difference in the temperature for samples of a different initial composition. Therefore, the dependence of diffusion coefficients on temperature is approximated to the dependence of diffusion coefficients on the initial sample composition $x$ :

$$
D=D^{\left(c_{\mathrm{Be}}=0\right)}(1-k \cdot x)
$$

The best fit results, shown as the lines in Fig. 4, are obtained at the values of following parameters: the efficient diffusion coefficient of beryllium in iron $D_{\mathrm{Be}}=2.9 \times 10^{-9} \mathrm{~m}^{2} \mathrm{~s}^{-1}$, when concentration of beryllium $c_{\mathrm{Be}} \rightarrow 0$ or for iron-rich samples, the dependence on composition coefficient $k=0.988$, and the ratio of diffusion coefficients $D_{\mathrm{Fe}} / D_{\mathrm{Be}}=0.66$. The value of the coefficient $k$ close to unity supports the conclusion that the average temperature for the beryllium-rich samples is lower and, therefore, the diffusion is much slower. The values of diffusion 
coefficients are average values calculated for the entire pulse duration. Nevertheless, the value of the efficient diffusion coefficient, $2.9 \times 10^{-9} \mathrm{~m}^{2} \mathrm{~s}^{-1}$, is much higher than the values known for the solid state [2] and it should be attributed to the efficient diffusion in the liquid state.

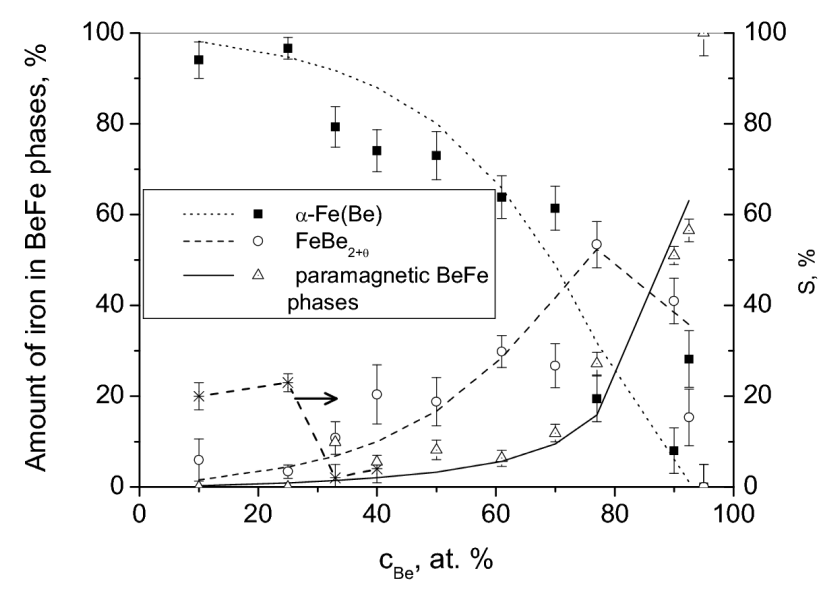

Fig. 4. Phase structure of samples obtained by means of Mössbauer spectroscopy and theoretical curves obtained by the fit of the diffusion model to experimental data. The right axis - the amount of iron in the products of the reaction with atmosphere.

The agreement of the phase structure experimentally found with diffusion modelling results (Fig. 4) is quite good. The discrepancies can arise due to the one-dimensional model that does not take into account nonuniform temperature. In addition, the real distribution of particles is three-dimensional and random.

\section{Results of the experiment and discussion}

The Mössbauer subspectra with the hyperfine magnetic field within 23-38 T (Fig. 2b), larger than the value for $\mathrm{FeBe}_{2}, 19.2 \pm 0.5 \mathrm{~T}$ [9], are attributed to beryllium solution in iron $\alpha-\mathrm{Fe}(\mathrm{Be})$, where the regions with the beryllium high and low concentrations can be observed [10]. Beryllium-rich or supersaturated ironberyllium solution can be in an ordered form as $\mathrm{Fe}_{3} \mathrm{Be}\left(\mathrm{DO}_{3}\right.$ ordering) or as $\mathrm{B} 2$ phase, where an atom percentage of beryllium can be $28-50$ at.\% [10, 11]. A wide distribution of the effective magnetic field in our samples (Fig. 2b), when $x>0.3$, is similar to that obtained in [10]. Therefore, it is expected that a considerable amount of beryllium in $\alpha$ - $\mathrm{Fe}(\mathrm{Be})$ solution can be found for the composition of $\mathrm{Be}_{x} \mathrm{Fe}_{1-x}$ samples when $0.3<x<0.6$. As the samples are produced by the laser, it is expected that after quenching a quite random distribution of beryllium atoms in $\alpha-\mathrm{Fe}(\mathrm{Be})$ solution is formed. For the description of Mössbauer spectra, two nearest coordination shells of an iron atom with the number $(m, n)$ of beryllium 
atoms are usually considered [10]. Then, the hyperfine magnetic field of an iron atom is given by

$$
H=H(0,0)+m \Delta H_{1}+n \Delta H_{2},
$$

where $\Delta H_{1}$ and $\Delta H_{2}$ are changes in the hyperfine magnetic field, when the $\mathrm{Be}$ atom replaces the Fe atom in the first and second coordination shells. A wide hyperfine field distribution $P(H)$ was obtained by us (thick line 1 in Fig. 2b II) using a binomial random distribution of Be atoms in the $\alpha$ - $\mathrm{Fe}(\mathrm{Be})$ solution and Eq. (6) when the beryllium concentration is uniformly distributed in its possible range in the $\alpha-\mathrm{Fe}(\mathrm{Be})$ solution, $0-50$ at. $\%$, and $\Delta H_{1}=2.6 \mathrm{~T}$ and $\Delta H_{2}=1.3 \mathrm{~T}$. As seen in Fig. 2b II, in addition to the region with uniform wide distribution of Be concentration, some extra region with beryllium low concentration in the $\alpha-\mathrm{Fe}(\mathrm{Be})$ solution is required.

When $x>0.5$, the samples are saturated with beryllium at a high concentration, and considerable amounts of beryllium-rich $\mathrm{FeBe}_{2,5,11}$ compounds are formed. For $0.6<x<0.8$, the presence of hyperfine magnetic fields is evident within 5-22 T. It indicates that $\mathrm{FeBe}_{2+\theta}$ is not stoichiometric and $\theta>0$. Different hyperfine magnetic fields were associated with the sites of iron atoms having the different number of iron atoms in nearest neighbour sites [9]. The largest hyperfine magnetic field of 19-20 $\mathrm{T}$ at room temperature is associated with a site having four nearest iron neighbours for the stoichiometric region of phase. When $\theta$ increases, the hyperfine magnetic field decreases. The enrichment of $\mathrm{FeBe}_{2+\theta}$ compound with beryllium causes a substitution of iron atoms in the iron sublattice. The probability of an iron atom to be surrounded by $m$ iron atoms in first neighbour sites is given by

$$
P(m)=\left(\begin{array}{c}
4 \\
m
\end{array}\right)(1-c)^{m} c^{4-m},
$$

where $c$ is beryllium concentration in the iron sublattice.

The subspectra used for description of spectra of the $\mathrm{Be}_{0.77} \mathrm{Fe}_{0.23}$ sample with the highest amount of $\mathrm{FeBe}_{2+\theta}$ are shown for the spectrum measured at $125 \mathrm{~K}$ (Fig. 3a). The results of spectra decomposition for $\mathrm{FeBe}_{2+\theta}$ phase are presented in Table I. Four areas of different compositions (different $\theta$ ) are used. The hyperfine magnetic field $H(m)$ of sextets (Table I) corresponds to the sites having 4,3 , and 2 nearest iron neighbours [9]. Since the hyperfine magnetic field values similar to those in [9] are used, the enrichment factor $\theta$ for $A, B$, and $C$ areas (Table I) should be $0,0.33$, and 0.63 , respectively. The region $D$ with $\theta \approx 1$ is added, because it allows us to have relative probabilities of subspectra more consistent with the values given by Eq. (7). A sufficiently good agreement between the values of probabilities for the spectra measured at room temperature and at $125 \mathrm{~K}$ supports the used model. Admittedly, the wide distribution of the composition parameter $\theta$ makes the determination of the probabilities of different surroundings difficult because of overlapping of subspectra lines. 
The compounds, cubic $\mathrm{FeBe}_{5}$ and hexagonal $\mathrm{FeBe}_{11}$, are paramagnetic and, by means of Mössbauer spectroscopy, can be determined by areas of singlets or quadrupole doublets at the centre of the Mössbauer spectrum. X-diffraction data help us to identify beryllium-rich compounds in the samples (Fig. 1). It has been found that there are cubic nonstoichiometric $\mathrm{FeBe}_{5-15}$ [12], stoichiometric $\mathrm{FeBe}_{5}$, hexagonal $\mathrm{FeBe}_{11}$, and residual Be. Some reaction products of beryllium with atmosphere $\left(\mathrm{BeO}, \mathrm{Be}_{3} \mathrm{~N}_{2}, \mathrm{Be}_{9} \mathrm{O}_{6} \mathrm{~N}_{2}\right.$ ) were found, whereas the oxidation of iron was not observed for the beryllium-rich samples. In the preliminary description of Mössbauer spectra (by distributions of hyperfine parameters), the paramagnetic part was described by the isomer shift distribution (Fig. 2c). Though the paramagnetic part for the $\mathrm{Be}_{0.77} \mathrm{Fe}_{0.23}$ sample (Fig. 2a IV), which contains about $20 \%$ of iron atoms, can be well expressed by a single doublet with the following parameters — the linewidth $\Gamma=0.32 \mathrm{~mm} \mathrm{~s}^{-1}$, the quadrupole splitting $\Delta=0.23 \mathrm{~mm} \mathrm{~s}^{-1}$ and the isomer shift $\delta=0.16 \mathrm{~mm} \mathrm{~s}^{-1}$ (all isomer shifts are referenced to metallic iron) - it does not indicate that only a single paramagnetic phase could be found. Analysis of composite Mössbauer spectra that contain nonstoichiometric compounds is difficult. In addition, as the data on beryllium-rich compounds $\mathrm{FeBe}_{5}, \mathrm{FeBe}_{11}$ are few, they really require a more detailed study.

The paramagnetic parts of Mössbauer spectra of the $\mathrm{Be}_{0.9} \mathrm{Fe}_{0.1}$ and $\mathrm{Be}_{0.95} \mathrm{Fe}_{0.05}$ samples contain approximately $50 \%$ and $100 \%$ of iron atoms (Fig. 3a). The spectra measured at low velocity (Fig. 3b) are slightly asymmetric, and, for their shape approximation, two quadrupole doublets are needed. The parameters of doublets are presented in Table II. A larger doublet has a slightly larger isomer shift $\delta$ and quadrupole splitting $\Delta$.

TABLE II

Mössbauer parameters of paramagnetic $\mathrm{Be}_{x} \mathrm{Fe}_{1-x}$ compounds.

\begin{tabular}{c|c|c|c|c|c}
\hline \hline$x$ & Subspectra & Area, $\%$ & $\delta, \mathrm{mm} \mathrm{s}^{-1}$ & $\Delta, \mathrm{mm} \mathrm{s}^{-1}$ & $\Gamma, \mathrm{mm} \mathrm{s}^{-1}$ \\
\hline 0.9 & 1 & $40 \pm 10$ & $0.14 \pm 0.02$ & $0.20 \pm 0.01$ & $0.32 \pm 0.01$ \\
& 2 & $60 \pm 10$ & $0.23 \pm 0.02$ & $0.24 \pm 0.01$ & $0.32 \pm 0.01$ \\
0.95 & 1 & $13 \pm 7$ & $0.13 \pm 0.02$ & $0.20 \pm 0.01$ & $0.32 \pm 0.01$ \\
& 2 & $87 \pm 7$ & $0.23 \pm 0.02$ & $0.26 \pm 0.01$ & $0.32 \pm 0.01$
\end{tabular}

Previously reported data on $\mathrm{FeBe}_{5}$ are the following: the quadrupole splitting $\Delta=0.25-0.29 \mathrm{~mm} \mathrm{~s}^{-1}$ and the isomer shift $\gamma=0.16-0.22 \mathrm{~mm} \mathrm{~s}^{-1}$ [13]. The quadrupole splitting $\Delta$ for iron impurity in beryllium at room temperature is $0.57 \mathrm{~mm} \mathrm{~s}^{-1}$ and the isomer shift $\delta=0.12 \mathrm{~mm} \mathrm{~s}^{-1}$ [14]. Evidently, on the basis of quadrupole splitting the paramagnetic part of beryllium-rich samples (Fig. 4) could mainly be attributed to cubic $\mathrm{FeBe}_{5}$ or nonstoichiometric analogue $\mathrm{FeBe}_{5-15}$. This conclusion is also confirmed by X-ray data (Fig. 1), where, for the sample composition with $x=0.925$, $\mathrm{X}$-ray lines of $\mathrm{FeBe}_{5-15}$ phase are most intense. Consequently, 
a larger doublet in Fig. $3 \mathrm{~b}$ is attributed to $\mathrm{FeBe}_{5-15}$ phase. The second doublet may be due to more iron-rich stoichiometric $\mathrm{FeBe}_{5}$ or due to the paramagnetic part of $\mathrm{FeBe}_{2+\theta}$ compound when $\theta$ is large. However, at low temperature, $T=125 \mathrm{~K}$, the increase in ferromagnetic part of $\mathrm{FeBe}_{2+\theta}$ for the sample $\mathrm{Be}_{0.77} \mathrm{Fe}_{0.23}$ is not observed, as could be expected if a considerable amount of paramagnetic $\mathrm{FeBe}_{2+\theta}$ is observed at room temperature. Attribution of paramagnetic part mainly to $\mathrm{FeBe}_{5-15}$ phase does not contradict the presence of a small amount of $\mathrm{FeBe}_{11}$ and the iron solution in beryllium because these compounds have a lower percentage of iron.

The value of the isomer shift for paramagnetic $\mathrm{FeBe}_{5}$ or $\mathrm{FeBe}_{2+\theta}$ can be explained by the Miedema theory [15-17]. According to the Miedema and van der Woude model $[16,17]$, the isomer shift of iron in the binary system $\mathrm{Fe}-\mathrm{A}(\mathrm{A}=\mathrm{Be})$ is

$$
\delta_{\mathrm{Fe}}\left(c_{\mathrm{Fe}}\right)=S_{\mathrm{A}}\left(c_{\mathrm{Fe}}\right)\left[0.75\left(\phi_{\mathrm{A}}-\phi_{\mathrm{Fe}}\right)-1.65\left(\frac{n_{\mathrm{A}}-n_{\mathrm{Fe}}}{n_{\mathrm{Fe}}}\right)\right]\left(\mathrm{mm} \mathrm{s}^{-1}\right),
$$

where $\phi$ is the electronegativity parameter, $n$ is the electron density at the boundary of the Wigner-Seitz cells of a pure element. For a solid solution, the surface area concentration of A surrounding $\mathrm{Fe}, S_{\mathrm{A}}$, is given by

$$
S_{\mathrm{A}}\left(c_{\mathrm{Fe}}\right)=\frac{\left(1-c_{\mathrm{Fe}}\right) V_{\mathrm{A}}^{2 / 3}}{c_{\mathrm{Fe}} V_{\mathrm{Fe}}^{2 / 3}+\left(1-c_{\mathrm{Fe}}\right) V_{\mathrm{A}}^{2 / 3}} .
$$

$V_{\mathrm{A}}$ and $V_{\mathrm{Fe}}$ are molar volumes. In the system with a strained structure the volume mismatch term,

$$
\delta_{v}=\frac{0.615 K_{\mathrm{A}}}{0.615 K_{\mathrm{A}}+K_{\mathrm{Fe}}} \frac{V_{\mathrm{A}}-V_{\mathrm{Fe}}}{V_{\mathrm{Fe}}} \frac{\partial(\delta)}{\delta(\ln V)},
$$

should be added, where $K$ is the bulk modulus. The most frequently used value of the isomer shift dependence on the volume $\partial(\delta) / \partial(\ln V)$, obtained from high pressure measurements, is $1.33 \mathrm{~mm} \mathrm{~s}^{-1}$ [16].

For a solid solution corresponding to the percentage of constituents of $\mathrm{FeBe}_{5}$ $\left(c_{\mathrm{Be}}=83\right.$ at.\%) the surface area concentration is $S_{\mathrm{A}}=0.79$. Using this value in Eq. (8), the value of the isomer shift $\delta=\delta_{\mathrm{Fe}}\left(c_{\mathrm{Fe}}\right)$ is $0.28 \mathrm{~mm} \mathrm{~s}^{-1}$. For ordered alloys and compounds, such as $\mathrm{FeBe}_{5}$, the surface area concentration is higher, and the isomer shift can approach the value of impurity of iron in beryllium. The analysis of X-ray spectra (Fig. 1) indicates that instead of stoichiometric cubic $\mathrm{FeBe}_{5}$ with a lattice constant of $5.889 \mathrm{~nm}$ the nonstoichiometric cubic $\mathrm{FeBe}_{5-15}$ is mainly observed. For $\mathrm{FeBe}_{5-15}$, the lattice constant is $5.863 \mathrm{~nm}$ when the concentration of iron is $c_{\mathrm{Fe}}=6.8$ at.\%, and it increases toward $5.889 \mathrm{~nm}$ when $c_{\mathrm{Fe}}$ approaches the stoichiometric value of 16.7 at.\% [12]. The lattice constant of $\mathrm{FeBe}_{5-15}$ is lower because the material has more beryllium atoms in the same structure as in $\mathrm{FeBe}_{5}$, and therefore the strain toward iron atoms similar to that described by Eq. (10) should arise. Applying the volume mismatch term $\delta_{v}=-0.24 \mathrm{~mm} \mathrm{~s}^{-1}$, the lower isomer shift limit $\delta=\delta_{\mathrm{Fe}}\left(c_{\mathrm{Fe}}\right)+\delta_{v}=0.04 \mathrm{~mm} \mathrm{~s}^{-1}$ is obtained. Therefore, 
the introduction of strain can explain the experimental isomer shift values of the paramagnetic part of Mössbauer spectra in our experiment. Similar conclusions on the isomer shift values, as for $\mathrm{FeBe}_{5-15}$, should be valid for paramagnetic and nonstoichiometric $\mathrm{FeBe}_{2+\theta}$. For $\mathrm{FeBe}_{2+\theta}$ compound the surface area concentration $S_{\mathrm{A}}$ is lower and, therefore, the isomer shift $\delta$ is less than that of $\mathrm{FeBe}_{5-15}$ phase. Additionally, $\mathrm{FeBe}_{11}$ compound could have the isomer shift very similar to that of $\mathrm{FeBe}_{5-15}$ compound.

\section{Conclusions}

Taking into account the dependence of the experimental conditions on the initial composition of the samples, the phase structure can be explained by mutual diffusion of constituent atoms in the molten or partly molten state. Efficient diffusion coefficients, that are averaged for the whole volume at a high temperature gradient and entire laser irradiation time, are higher than they could be obtained for solid state. The analysis of Mössbauer spectra parameters has shown that the obtained $\mathrm{FeBe}_{2+\theta}$ and $\mathrm{FeBe}_{5}$ phases are nonstoichiometric and strained. The composition of $\mathrm{FeBe}_{2+\theta}$ and $\mathrm{FeBe}_{5}$ compounds varies in a wide interval.

\section{References}

[1] A.F. Jankowski, M.A. Wall, A.W. Van Buuren, T.G. Nieh, J. Wadsworth, Acta Materialia 50, 4791 (2002).

[2] K.K. Kadyrzhanov, V.S. Rusakov, T.E. Turkebaev, E.A. Kerimov, A.D. Lopuga, Nucl. Instrum. Methods B 174, 463 (2001).

[3] K.K. Kadyrzhanov, V.S. Rusakov, T.E. Turkebaev, M.F. Vereschak, E.A. Kerimov, D.A. Plaksin, Hyperfine Interact. 141/142, 453 (2002).

[4] A. Amulevicius, M. Balciuniene, Br. Petretis, R. Pileckis, Thin Solid Films 229, 192 (1993).

[5] A. Amulevicius, M. Balciuniene, Br. Petretis, R. Pileckis, Thin Solid Films 240, 60 (1994).

[6] W.H. Press, S.A. Teukolsky, W.T. Vetterling, B.P. Flannery, Numerical Recipes in Fortran 77, Vol. 1, 2nd ed., Cambridge University Press, New York 1993, p. 670.

[7] J.Y. Ping, D.G. Rancourt, Hyperfine Interact. 92, 1209 (1994).

[8] R.W. Smith, X. Zhu, M.C. Tunnicliffe, T.J.N. Smith, L. Misener, J. Adamson, Ann. New York Acad. Sci. 974, 57 (2002).

[9] K. Ohta, J. Appl. Phys. 39, 2123 (1968).

[10] K. Yagisawa, Phys. Status Solidi A 18, 589 (1973).

[11] M.K. Miller, M.G. Burke, S.S. Brenner, Scr. Metall. 18, 285 (1984).

[12] R. Teitel, M. Cohen, J. Met. 1, 285 (1949).

[13] S. Gupta, K.B. Lal, T.M. Srinivasan, G.N. Rao, Phys. Status Solidi A 22, 707 (1974). 
[14] C. Janot, P. Delcroix, M. Piecuch, Phys. Rev. B 10, 2661 (1974).

[15] A.R. Miedema, J. Less-Common Metals 32, 117 (1973).

[16] C.L. Chien, G. Xiao, K.M. Unruh, Phys. Rev. B 32, 5582 (1985).

[17] A.R. Miedema, F. van der Woude, Physica B 100, 145 (1980). 